\title{
BMJ Open Psychometric properties of disease- specific health-related quality of life (HRQoL) instruments for food allergy and food intolerance: protocol for a COSMIN-based systematic review
}

Qirong Chen (D) ,1,2 Zeen Li, ${ }^{1}$ Aimee Castro (D) , ${ }^{3}$ Siyuan Tang (D) ,1,2 Jiarui Chen, ${ }^{1,2}$ Chongmei Huang, ${ }^{1}$ Jinnan Xiao (D) , ${ }^{1}$ Haiyan Liu, ${ }^{4}$ Jinfeng Ding (D) ${ }^{1,2}$

To cite: Chen Q, Li Z,

Castro A, et al. Psychometric properties of disease-specific health-related quality of life (HRQoL) instruments for food allergy and food intolerance: protocol for a COSMIN-based systematic review. BMJ Open 2022;12:e053534. doi:10.1136/ bmjopen-2021-053534

- Prepublication history and additional supplemental material for this paper are available online. To view these files, please visit the journal online (http://dx.doi.org/10.1136/ bmjopen-2021-053534).

Received 15 May 2021 Accepted 19 November 2021

Check for updates

(C) Author(s) (or their employer(s)) 2022. Re-use permitted under CC BY-NC. No commercial re-use. See rights and permissions. Published by BMJ.

For numbered affiliations see end of article.

Correspondence to Dr Jinfeng Ding; jinfeng.ding@csu.edu.cn

\section{ABSTRACT}

Introduction Food allergies and food intolerances can bring burdens on patients and their caregivers and reduce health-related quality of life (HRQLL). An increasing number of disease-specific HRQoL instruments for food allergies and food intolerances has been developed, and some of them have been adapted for different cultures and languages. This report describes a protocol for a systematic review of the psychometric properties of these instruments. The aims of this systematic review are to: (1) formulate recommendations for the usage of existing validated disease-specific HRQoL instruments for patients with food allergies and/or food intolerances and their caregivers; and (2) identify knowledge gaps to inform future research relating to these instruments.

Methods and analysis This protocol adheres to the Preferred Reporting Items for Systematic Reviews and Meta-Analyses Protocol (PRISMA-P) 2015 checklist. The future review will follow the Consensus-based Standards for the Selection of Health Measurement Instruments (COSMIN) guideline for systematic reviews of patientreported outcome measures (PROMs) and PRISMA 2020 statement guideline. Six databases (PubMed, EMBASE, Web of Science, Scopus, CINAHL and ProQuest -Health \& Medical Collection) will be searched to retrieve studies focusing on the development and psychometric properties of disease-specific HRQoL instruments for patients with food allergies and/or food intolerances and their caregivers between 1 December 2021 and 31 December 2021. Two researchers will be responsible for literature screening, data extraction and literature evaluation, independently. Disagreements will be addressed by discussion or the involvement of a third researcher. The methodological quality of the included studies and the quality of the identified instruments will be assessed based on the COSMIN guideline for systematic reviews of PROMs.

Ethics and dissemination Ethical approval is not applicable for this study. We will disseminate the findings through publication in peer-reviewed journals and/or academic conferences.

PROSPERO registration number CRD42021252203.

\section{Strengths and limitations of this study}

- To our knowledge, this is the first Consensus-based Standards for the Selection of Health Measurement Instruments (COSMIN)-based systematic review of disease-specific health-related quality of life (HRQoL) instruments for food allergies and food intolerances.

- All up-to-date specific guidelines (Preferred Reporting Items for Systematic Reviews and MetaAnalyses (PRISMA) Protocol 2015 checklist, updated COSMIN guideline for systematic reviews of patientreported outcome measures (PROMs) and PRISMA 2020 checklist) will be used to guide the implementation and report of the protocol and systematic review.

- The COSMIN guideline for systematic reviews of PROMs will be used to evaluate the methodological quality of included studies on measurement properties of the instruments and the quality of included instruments.

- The systematic review may fail to include relevant literature that were not included in the searched databases.

\section{INTRODUCTION}

Adverse reactions to food encompass food allergies and food intolerances. ${ }^{1}$ Food allergies and food intolerances are common, especially in children, with $6.25 \%-28.0 \%$ of children having had this experience. ${ }^{1}$ Food allergies and food intolerances have become severe public health problems worldwide. ${ }^{2} \mathrm{~A}$ food allergy is an abnormal immunological reaction to specific food(s) that results in the development of symptoms. ${ }^{3}$ Food allergies include three types of immunological reactions: IgE-mediated, non-IgE-mediated and Mixed IgE- and non-IgE-mediated. ${ }^{1}$ Food intolerances are non-immune mediated adverse reactions to food; lactose intolerance 
is one such example. ${ }^{1}$ Food allergies and food intolerances impact multiple organs and systems, and are associated with a range of symptoms (eg, urticaria, eczema, colic, vomiting, reflux, diarrhoea or constipation, blood in stool and growth retardation). ${ }^{13}$ Meanwhile, food allergies may lead to life-threatening anaphylaxis. ${ }^{4}$

Adverse food intake reactions bring great challenges to healthcare, education, food and catering industries in many countries. Critically, the physiological, psychological, social and financial burdens relating to food allergies and food intolerances also undermine health-related quality of life (HRQoL) of patients and their family caregivers. ${ }^{5}$ Instruments measuring HRQoL can help quantify the impacts of food allergies and food intolerances, and may support better prevention and management of this problem. For example, healthcare professionals could use these instruments to assess the quality of life of patients and their caregivers to provide specific healthcare services and suggestions. Furthermore, researchers can use these instruments in studies to evaluate the quality of life of patients and their caregivers.

There are two types of HRQoL instruments: generic and disease-specific. ${ }^{5}$ Disease-specific HRQoL instruments are more likely to have a higher level of sensitivity compared with generic HRQoL instruments. ${ }^{56}$ A number of disease-specific HRQoL instruments for food allergies has been developed. Some of them have been validated and adapted into multiple versions for different cultures and languages. ${ }^{6}$ Since 2014, there have also been some studies reporting on the overall development of instruments for food intolerances. ${ }^{8-10}$ Two literature reviews relating to HRQoL instruments for food allergies were published in 2009 and 2014, respectively. ${ }^{6}$ The 2009 review summarised and described generic and diseasespecific instruments for food allergies in children and adults. ${ }^{6}$ However, this review was limited by its use of a narrative review approach, rather than a systematic review following corresponding guidelines, which can lead to the omission of some important literature. The 2014 review systematically summarised and evaluated all disease-specific HRQoL instruments for IgE-mediated food allergies. ${ }^{7}$ However, this systematic review failed to include other types of food allergies (non-IgE-mediated, and Mixed IgE- and non-IgE-mediated), as well as food intolerances. These omiited types of adverse reactions to food also have significant influences on quality of life of patients and caregivers, and as such, should be included in a broader systematic review of the literature. Furthermore, the 2014 review did not follow specific guidelines for evaluating the methodological quality of the included studies and the quality of the included instruments. In 2018, the Consensus-based Standards for the Selection of Health Measurement Instruments (COSMIN) guideline was developed for systematic reviews of Patient-Reported Outcome Measures (PROMs). ${ }^{11-14}$ This guideline could improve the quality of the systematic review of PROMs, offering researchers a critical and comprehensive evaluation of the available instruments.
Therefore, the overall aim of this systematic review is to critically describe, appraise, and summarise the existing disease-specific HRQoL instruments for patients with food allergies and/or food intolerances and their family caregivers, based on the COSMIN guideline for systematic reviews of PROMs. ${ }^{13}$ The specific objectives of the proposed systematic review are to: (1) identify and describe all existing validated disease-specific HRQoL instruments for patients with food allergies and/or food intolerances and their caregivers; (2) evaluate the methodological quality of studies on measurement properties of the instruments and (3) assess and compare the psychometric properties and other key characteristics of these instruments.

This systematic review will answer the following questions: (1) What are existing disease-specific HRQoL instruments for patients with food allergies and/or food intolerances and their caregivers? (2) What are the characteristics of these instruments? (3) What is the methodological quality of studies on measurement properties of these instruments? (4) What are the measurement properties, interpretability and feasibility of these instruments? (5) What are the similarities and differences among these instruments? and (6) What are the knowledge and research gaps in this area?

\section{METHODS}

This is a protocol for a systematic review following Preferred Reporting Items for Systematic Reviews and Meta-Analyses Protocols (PRISMA-P) 2015 checklist. ${ }^{15}$ Based on COSMIN guideline for systematic reviews of PROMs, we adapted the 'Objectives' section of the PRISMA-P 2015 checklist by replacing 'Participants, Interventions, Comparators and Outcomes' with 'Construct, Population(s), Type of Instrument(s) and Measurement properties'. ${ }^{11}$ We registred the protocol in the International Prospective Register of Systematic Reviews (PROSPERO), and the title of the protocol has been registered on the Joanna Briggs Institute website. We will conduct this systematic review based on the COSMIN guideline for systematic reviews of PROMs ${ }^{13}$ and report it following the updated PRISMA 2020 checklist. ${ }^{16}$

\section{Inclusion and exclusion criteria of studies \\ Inclusion criteria}

Studies will be included if they: (1) report disease-specific HRQoL instrument(s) designated for patients with food allergies and/or food intolerances, and/or their caregivers; (2) describe the processes of development and/ or evaluation of one or more measurement properties for eligible instrument(s) and (3) have full-text availability. The authors of the articles will be approached if a full-text version is not available online. However, if the authors' contact information is not available or the authors do not respond to the inquiry, these studies will be excluded but their information will be recorded in online supplemental file of the formal systematic review. 


\section{Exclusion criteria}

Studies will be excluded if they: (1) are not primary studies (eg, discussion papers, letters and editorials) or case studies or (2) are reports that used the instruments only for outcome measurement.

\section{Search strategy}

Between 1 December 2021 and 31 December 2021, we will search PubMed, EMBASE, Web of Science, Scopus, CINAHL and ProQuest (Health \& Medical Collection) using comprehensive and sensitive search strategies that combine Medical Subject Heading and free text words. All databases will be searched from the date of inception to the date of searching. The major search concepts will be quality of life (Construct), food allergies/food intolerances (Population), PROMs (Type of instrument(s)) and measurement properties. Three comprehensive and sensitive keyword search strategies developed by other researchers for reviews of the concepts will be used in this literature search. They are: (1) the search filter of 'quality of life' for medical and health bibliographic databases developed by Dutch medical information specialists ${ }^{17}$; (2) the search filter for finding PROMs developed by the University of Oxford $^{18}$; (3) the sensitive PubMed search filter for measurement properties developed by Terwee et $a l$, and corresponding search filters adapted for other databases. These literature search filters will improve the comprehensiveness, effectiveness and quality of the literature search in this study. ${ }^{19}$ Furthermore, a health science librarian had been consulted for developing the search strategies. Online supplemental table S1 and online supplemental tables S9-S13 show the search strategies we developed for the databases searched. The search will not be limited to a specific language; that is, we will include eligible publications in any language, and a translation service will be used if needed. Database searches will be carried out again to provide a final update of the searches after the systematic review is accepted by a journal. The systematic review will be updated if new eligible studies are identified.

\section{Study screening}

Endnote and Covidence will be used to manage the references screening. First, we will use EndNote to recognise and remove duplicates, and then conduct manual screening. ${ }^{20}$ Following this initial screening, titles, abstracts and full-text articles will be reviewed and screened independently by two researchers with the support of Covidence. Disagreements between the two researchers will be addressed through discussion. Consultation of a third researcher will be adopted where necessary. Reference lists from all eligible papers will also be screened using the aforementioned inclusion and exclusion criteria. The processes of study screening are shown in figure 1 .

\section{Data extraction}

Two researchers will conduct the data extraction independently. A third researcher will review the extracted data and address the discrepancies between the two researchers if identified. We will extract data on (1) basic characteristics of the included instruments (online supplemental table S2, including: the name of the instrument, developer(s)/year developed, construct(s), targeted population, mode of administration, recall period, (sub) scale (s)/ (number of items), response options, range of scores/scoring, original language and available translations); (2) characteristics of the included study populations (online supplemental table S3, including sample size, age, gender, disease, disease duration and severity, setting, country, language); (3) results of measurement properties of the included instruments (Result columns in online supplemental table S5, including content validity, structural validity, internal consistency, crosscultural validity, measurement invariance, reliability, measurement error, criterion validity, construct validity, responsiveness and (4) interpretability (online supplemental table S7) and feasibility (online supplemental table S8) of the included instruments.

\section{Quality appraisal and data synthesis}

Two researchers will conduct the quality assessment for included studies and instruments independently. A third researcher will be consulted if consensus could not be reached. The COSMIN guideline will be used to assess each subscale of a multidimensional PROM separately. ${ }^{21}$ Therefore, the measurement properties for subscale scores and the entire PROM will be rated separately in this study.

In the first step, COSMIN standards for design requirements and preferred statistical methods will guide the evaluation of the methodological quality of the included studies on the development and measurement properties of the instruments. ${ }^{11} 1314$ The following COSMIN resources will be used in this phase: the COSMIN Risk of Bias checklist for PROMs, ${ }^{12}$ the COSMIN methodology for systematic reviews of PROMs-User manual, ${ }^{11}$ and the COSMIN methodology for assessing the content validity of PROMs-User manual. ${ }^{22}$ In this step, an Excel sheet file named 'Scoring form COSMIN boxes' will be used to manage the evaluation records (refer to online supplemental additional file 3 ; this file is also available at https:/ / www.cosmin.nl/tools/guideline-conducting-systematic-review-outcome-measures/); this file is provided by the COSMIN guideline. The final consensus on the results of the methodological quality will be presented in online supplemental table S4 and Meth qual column in online supplemental table S5.

In the second step, we will evaluate the results associated with measurement properties of identified instruments according to the COSMIN quality criteria for good measurement properties. ${ }^{21} 23$ The corresponding results will be reported in the rating columns in online supplemental table S5. However, the rating results of content validity will be separately presented in online supplemental table S5-1 given that criteria and rating systems 


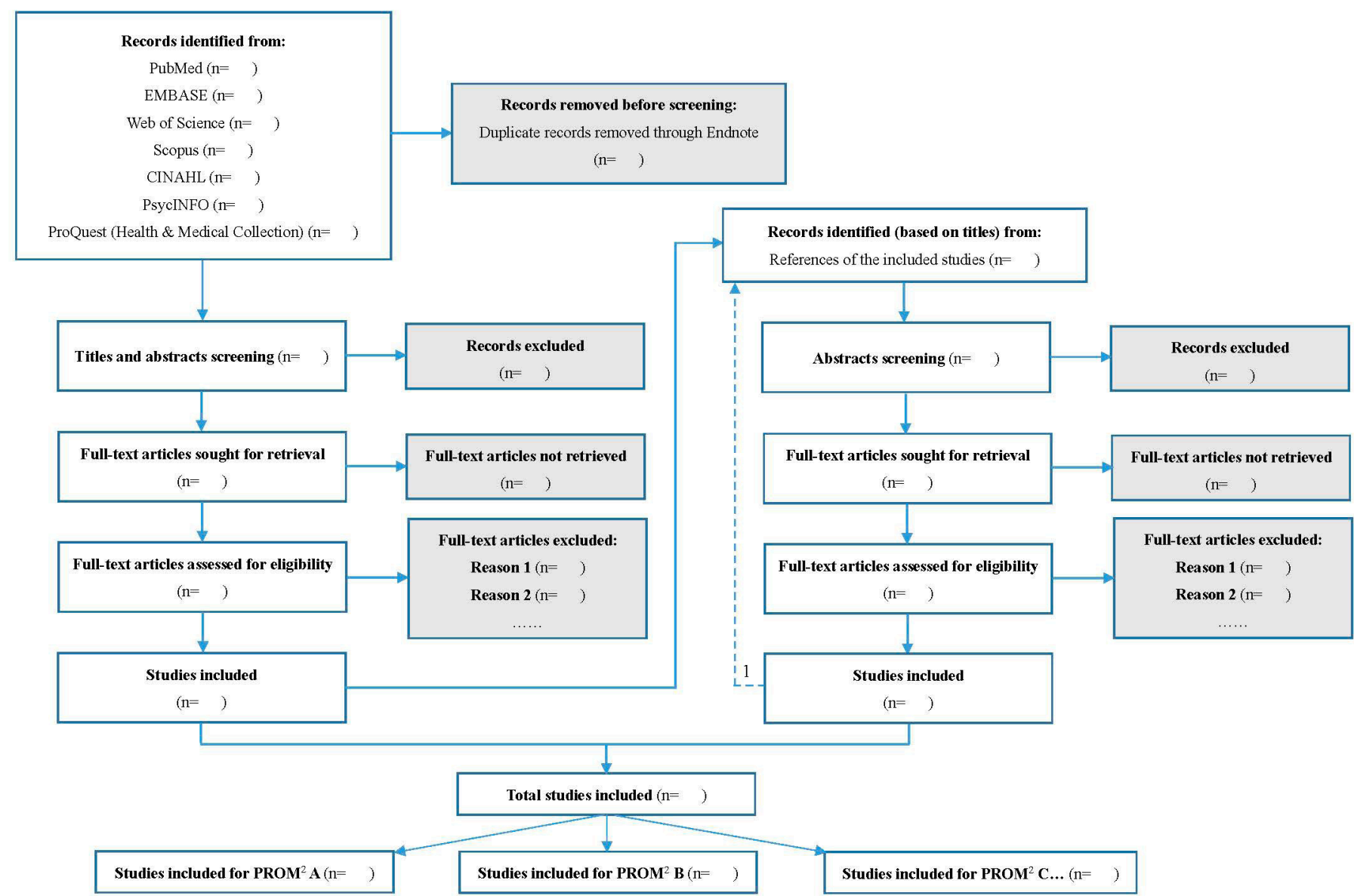

Figure 1 Flow chart of literature selection process. PROM, patient-reported outcome measure.

for evaluation of content validity of PROMs differ from other measurement properties in COSMIN guideline. ${ }^{23}$

The COSMIN guideline also provides a separate table (online supplemental table S6) to synthesise evidence and results associated with measurement properties. In the third step, we will statistically pool or qualitatively summarise the results on measurement properties from different studies providedand show the summarised or pooled results in the column of Summary or pooled results of online supplemental table S6. A meta-analysis approach (weighted means and 95\% CIs) will be used when possible. We will evaluate the pooled or summarised results per measurement property for each PROM according to the COSMIN quality criteria for good measurement properties; the corresponding results will be shown in the Overall rating columns in online supplemental table S6.

Finally, we will use the modified GRADE (Grading of Recommendations Assessment, Development and Evaluation) approach developed by the COSMIN guideline for systematic reviews of PROMs to grade the quality of the evidence. The quality of the evidence will be defined as the level of confidence based on the level of trustworthiness of the pooled or summarised result (shown in Quality of evidence columns in online supplemental table S6). The COSMIN guideline classifies the quality of the evidence into four levels: 'high', 'moderate', 'low' or 'very low'. ${ }^{21}$
These findings will enable us to formulate recommendations on the usage of existing disease-specific HRQoL instruments for patients with food allergies and/or food intolerances and their caregivers. Our findings will also identify knowledge gaps in this area and inform future research.

\section{Patient and public involvement}

Neither patients nor the public will be involved in this study.

\section{Ethics and dissemination}

Ethical approval is not applicable for this study. We will share the findings from the study at national and/or international conferences and in a peer-reviewed journal in the fields of food allergies or food intolerances.

\section{DISCUSSION}

To our knowledge, this review will be the first PRISMA and COSMIN guidelines-guided systematic review of diseasespecific HRQoL instruments for patients with food allergies and/or food intolerances and their caregivers. This review will identify, describe, evaluate and compare all eligible instruments. The methodological quality of all included studies on the measurement properties of these instruments and the psychometric properties of 
all included instruments will be evaluated based on the COSMIN guideline for systematic reviews of PROMs. The findings will facilitate the formulation of the recommendations on the usage of the targeted instruments for clinical practice and research. We will also identify knowledge gaps associated with measurements of HRQoL for patients with food allergies and/or food intolerances and their caregivers. This review has the potential to clearly identify opportunities for further research, and therefore supports future studies on the development and improvement of disease-specific HRQoL instruments for these populations.

\section{Author affiliations}

${ }^{1}$ Xiangya School of Nursing, Central South University, Changsha, Hunan, China ${ }^{2}$ Xiangya Center for Evidence-Based Practice \& Healthcare Innovation: A Joanna Briggs Institute Affiliated Group, Central South University, Changsha, Hunan, China ${ }^{3}$ Ingram School of Nursing, McGill University, Montreal, Quebec, Canada

${ }^{4}$ Third Xiangya Hospital, Central South University, Changsha, Hunan, China

\section{Twitter Aimee Castro @AimeeRCastro}

Contributors Study design: QC, JD and ST; Study supervision: JD, ST and HL; Manuscript writing: QC, ZL, JD and AC; Critical revisions for important intellectual content: AC, JC, CH, JX and $\mathrm{HL}$.

Funding The authors have not declared a specific grant for this research from any funding agency in the public, commercial or not-for-profit sectors.

Competing interests None declared.

Patient consent for publication Not applicable.

Provenance and peer review Not commissioned; externally peer reviewed.

Supplemental material This content has been supplied by the author(s). It has not been vetted by BMJ Publishing Group Limited (BMJ) and may not have been peer-reviewed. Any opinions or recommendations discussed are solely those of the author(s) and are not endorsed by BMJ. BMJ disclaims all liability and responsibility arising from any reliance placed on the content. Where the content includes any translated material, BMJ does not warrant the accuracy and reliability of the translations (including but not limited to local regulations, clinical guidelines, terminology, drug names and drug dosages), and is not responsible for any error and/or omissions arising from translation and adaptation or otherwise.

Open access This is an open access article distributed in accordance with the Creative Commons Attribution Non Commercial (CC BY-NC 4.0) license, which permits others to distribute, remix, adapt, build upon this work non-commercially, and license their derivative works on different terms, provided the original work is properly cited, appropriate credit is given, any changes made indicated, and the use is non-commercial. See: http://creativecommons.org/licenses/by-nc/4.0/.

\section{ORCID iDs}

Qirong Chen http://orcid.org/0000-0003-1908-290X

Aimee Castro http://orcid.org/0000-0002-6461-0866

Siyuan Tang http://orcid.org/0000-0001-9940-5072

Jinnan Xiao http://orcid.org/0000-0003-2403-8495

Jinfeng Ding http://orcid.org/0000-0002-8783-8919

\section{REFERENCES}

1 Manuyakorn W, Tanpowpong P. Cow milk protein allergy and other common food allergies and intolerances. Paediatr Int Child Health 2019;39:32-40.

2 Hossny E, Ebisawa M, El-Gamal Y, et al. Challenges of managing food allergy in the developing world. World Allergy Organ $J$ 2019;12:100089.
3 Muraro A, Werfel T, Hoffmann-Sommergruber K, et al. EAACl food allergy and anaphylaxis guidelines: diagnosis and management of food allergy. Allergy 2014;69:1008-25.

4 Muraro A, Roberts G, Worm M, et al. Anaphylaxis: guidelines from the European Academy of allergy and clinical immunology. Allergy 2014;69:1026-45.

5 Dunn Galvin A, Hourihane J O'B. Health-related quality of life in food allergy : Impact, correlates, and predictors. Bundesgesundheitsblatt Gesundheitsforschung Gesundheitsschutz 2016;59:841-8.

6 Flokstra-de Blok BMJ, Dubois AEJ. Quality of life in food allergy: valid scales for children and adults. Curr Opin Allergy Clin Immunol 2009;9:214-21.

7 Salvilla SA, Dubois AEJ, Flokstra-de Blok BMJ, et al. DiseaseSpecific health-related quality of life instruments for IgE-mediated food allergy. Allergy 2014;69:834-44.

8 DunnGalvin A, Barnett J, Begen FM, et al. Development and preliminary validation of the food intolerance quality of life questionnaire (FIQLQ): adult form. Qual Life Res 2018;27:1109-16.

9 Hourihane JO, Greenhawt MJ, Galvin AD. A new valid and reliable parent proxy questionnaire to measure the impact of food protein enterocolitis syndrome on children: the Fpies quality of life questionnaire, parent form. J Allergy Clin Immunol 2015;135:AB280.

10 Greenhawt MJ, Schultz F, Galvin AD. Caregiver quality of life in food protein enterocolitis syndrome. J Allergy Clin Immunol 2015;135:AB280.

11 Mokkink LB, Prinsen CAC, Patrick DL, et al. COSMIN methodology for systematic reviews of Patient-Reported Outcome Measures (PROMs) - user manual Netherlands: COSMIN, 2018. Available: https://cosmin.nl/wp-content/uploads/COSMIN-syst-review-forPROMs-manual_version-1_feb-2018.pdf [Accessed 5 May 2021].

12 Mokkink LB, de Vet HCW, Prinsen CAC, et al. COSMIN risk of bias checklist for systematic reviews of patient-reported outcome measures. Qual Life Res 2018;27:1171-9.

13 Prinsen CAC, Mokkink LB, Bouter LM, et al. COSMIN guideline for systematic reviews of patient-reported outcome measures. Qual Life Res 2018;27:1147-57.

14 Terwee CB, Prinsen CAC, Chiarotto A, et al. COSMIN methodology for evaluating the content validity of patient-reported outcome measures: a Delphi study. Qual Life Res 2018;27:1159-70.

15 Shamseer L, Moher D, Clarke M, et al. Preferred reporting items for systematic review and meta-analysis protocols (PRISMA-P) 2015 elaboration and explanation. BMJ 2015;350:g7647.

16 Page MJ, Moher D, Bossuyt PM, et al. PRISMA 2020 explanation and elaboration: updated guidance and exemplars for reporting systematic reviews. BMJ 2021;372:n160.

17 Vissers T, Vries RD. Quality of life (QOL) search block Amsterdam: Afdeling Biomedische Informatiespecialisten; 2020, 2020. Available: https://blocks.bmi-online.nl/catalog/294 [Accessed 5 May 2021].

18 Mackintosh A, Comabella CCI, Hadi M, et al. PROM GROUP CONSTRUCT \& INSTRUMENT TYPE FILTERS Oxford: University of Oxford; 2010, 2010. Available: https://cosmin.nl/wp-content/uploads/ prom-search-filter-oxford-2010.pdf [Accessed 5 May 2021].

19 Terwee CB, Jansma EP, Riphagen II, et al. Development of a methodological PubMed search filter for finding studies on measurement properties of measurement instruments. Qual Life Res 2009;18:1115-23.

20 Bramer WM, Giustini D, de Jonge GB, et al. De-duplication of database search results for systematic reviews in endnote. J Med Libr Assoc 2016;104:240-3.

21 Mokkink LB, Prinsen CA, Patrick DL, et al. COSMIN methodology for systematic reviews of patient-reported outcome measures (PROMs) - user manual Amsterdam, the Netherlands COSMIN, 2018a. Available: https://cosmin.nl/wp-content/uploads/COSMINsyst-review-for-PROMs-manual_version-1_feb-2018.pdf [Accessed 27 Apr 2021]

22 Terwee CB, Prinsen CA, Chiarotto A, et al. COSMIN methodology for assessing the content validity of PROMs - user manual Netherlands: COSMIN; 2018, 2018. Available: https://cosmin.nl/wp-content/ uploads/COSMIN-methodology-for-content-validity-user-manual-v1. pdf [Accessed 5 May 2021].

23 Terwee CB, Prinsen CA, Chiarotto A, et al. COSMIN methodology for assessing the content validity of PROMs - User manual Amsterdam, The Netherlands: COSMIN, 2018b. Available: https://cosmin.nl/wpcontent/uploads/COSMIN-methodology-for-content-validity-usermanual-v1.pdf [Accessed 27 Apr 2021] 